\title{
ANÁLISIS DE RELATOS DE ESTUDIANTES UNIVERSITARIOS SOBRE LA AUTORREGULACIÓN EN EXPERIENCIAS DE EVALUACIÓN FORMATIVA
}

Analysis of university student experiences about self-regulation in formative evaluation

Análise das histórias de estudantes universitários sobre a autorregulação em

experiências de avaliação formativa

\author{
Txell Zolle-Valero Setó (1)
}

Marta Fuentes Agustí (2)

Aportación derivada del proyecto: “Estratègies d'autoavaluació per a l'autoregulació d'aprenentatges i el desenvolupament professional docent” (Ref.: 2017ARMIF 00006) financiado por AGAUR.

(1) Universidad Autónoma de Barcelona, España. Teléfono: +34 935812627. Correo electrónico: txell.zollevalero@,uab.cat

(2) Universidad Autónoma de Barcelona, España. Teléfono: +34 935812627. marta.fuentes@uab.cat

\section{Resumen}

El proceso de autorregulación del aprendizaje en la evaluación formativa universitaria implica que el estudiante controle su actividad y organice su entorno de acuerdo con unos objetivos académicos. El propósito del presente estudio es identificar prácticas de autorregulación relacionadas con procesos de aprendizaje. El método seguido es descriptivo-comprensivo basado en el análisis de relatos aportados por estudiantes de los Grados de Educación Infantil y Primaria impartidos en distintas Universidades. Los resultados indican que parte del proceso de autorregulación se da de manera inconsciente. Las pautas de control más explicitadas se dan en las primeras fases del desarrollo de las tareas. Se identifican seis elementos clave del constructo, entre ellos resalta la incidencia positiva de los instrumentos y comentarios de retroacción facilitados por los docentes. Se concluye con la necesidad de definir estrategias que promuevan la práctica auto-reguladora del aprendizaje de manera que luego se transfiera en la futura profesión educativa.

Palabras clave: Evaluación formativa; autorregulación; estudiante; educación superior; aprendizaje

Análisis de relatos de estudiantes universitarios sobre la autorregulación en experiencias de evaluación 


\begin{abstract}
The process of self-regulation of learning in the university formative evaluation implies that the student controls his activity and organizes his environment in accordance with some academic objectives. The purpose of this study is to identify self-regulation practices related to learning processes. The method followed is descriptivecomprehensive based on the analysis of experiences written by students of the Degree in Infant Education and the Degree in Primary Education taught in different universities. The results show that part of the self-regulation process occurs unconsciously. The most explicit control guidelines are found in the early stages of task development. Six key elements of the construct are identified, including the positive impact of the instruments and feedback provided by teachers. Findings show the need to define strategies that promote the self-regulating practice of learning so that it is later transferred in the future educational profession.
\end{abstract}

Keywords: Formative evaluation; self-regulation; student; higher education; learning.

\title{
Resumo
}

O processo de autorregulação da aprendizagem na avaliação formativa universitária implica que o aluno controle a sua atividade e organize o seu ambiente, de acordo com os objetivos académicos. O objetivo deste estudo é identificar práticas de autorregulação relacionadas com processos de aprendizagem. O método seguido é descritivoabrangente, baseado na análise de histórias proporcionadas por estudantes das Licenciaturas de educação pré-escolar e primária ministradas em diferentes universidades. Os resultados indicam que parte do processo de autorregulação ocorre inconscientemente. As diretrizes de controlo mais explícitas são dadas nas fases iniciais do desenvolvimento das tarefas. Identificam-se seis elementos chave do constructo, destacando-se entre eles, o impacto positivo dos instrumentos e os feedbacks dados pelos professores. Conclui-se com a necessidade de definir estratégias que promovam a prática autorreguladora de aprendizagem, de forma a que, posteriormente, se transfira para a futura profissão educativa.

Palavras-chave: Avaliação formativa; autorregulação; estudante; ensino superior; aprendizagem

Análisis de relatos de estudiantes universitarios sobre la autorregulación en experiencias de evaluación 


\section{Introducción}

La evaluación formativa (López-Pastor \& Pérez-Pueyo, 2017) implementada en la universidad para promover el aprendizaje de los contenidos propios de la profesión (Fuentes, 2017), la adquisición de competencias a lo largo de la vida (Cano, 2014) y el aprendizaje autónomo (Villardón, 2006) también propicia el desarrollo de procesos de autorregulación (Nicol \& Macfarlane, 2006; Andrade, 2010). La autorregulación del aprendizaje es un constructo psicológico que según Hernández y Camargo (2017, p.147) "Consiste en la organización deliberada de actividades cognitivas, conductuales y ambientales que conducen al éxito en el aprendizaje." Entre estas actividades es imprescindible la retroalimentación (Ion, Sánchez-Martí, Agud-Morell, 2019),

Ante la necesidad de estudios empíricos que analicen el desarrollo de la capacidad autoreguladora (Panadero, 2017) nace el presente proyecto con el objetivo de conocer y aportar evidencias sobre la implementación de metodologías docentes para la mejora de la formación inicial de los futuros maestros de Educación Infantil y Primaria. En concreto, este texto se centra en la exploración de la percepción del alumnado sobre la aplicación de estrategias de autorregulación en su desarrollo de aprendizaje formal, con la finalidad de llegar a sistematizar, en posteriores fases del proyecto, algunas estrategias para el aprendizaje de la autorregulación.

\section{Método}

El presente estudio descriptivo-comprensivo forma parte del primer objetivo de un estudio más amplio. Pretende identificar y analizar los factores que inciden en los procesos de autorregulación de los aprendizajes de los maestros en formación y en activo. En concreto se sitúa en la segunda actividad de la primera fase (A2. Identificación de prácticas de autorregulación relacionadas con procesos de aprendizaje y profesionales) donde se analiza mediante narrativas la percepción de los estudiantes sobre el conocimiento y la aplicación de estrategias de autorregulación en su proceso de aprendizaje. Los relatos analizados se construyeron a través de entrevistas estructuradas realizadas a inicios del 2019 en los propios entornos de formación, a una muestra no aleatoria intencional de 11 estudiantes, de entre $1^{\circ}$ y $4^{\circ}$ del Grado Educación Infantil y el Grado de Educación Primaria, de tres universidades catalanas. Las transcripciones de las entrevistas fueron revisadas por los estudiantes. Los textos finales fueron anonimizados, codificados y analizados con la ayuda del programa MAXQDA. 


\section{Resultados y discusión}

Se han identificado entre los relatos de los estudiantes seis elementos clave relacionados con el proceso de autorregulación del aprendizaje:

1. La retroalimentación que dan los profesores a los estudiantes durante el proceso o al final de las actividades lo consideran muy importante. Aunque hay quién dice que esta se limita a menudo a una calificación numérica por lo que no resulta ser una ayuda para la mejora. (FEEDBACK-DOCENTE)

2. El interés por el tema central de la tarea solicitada y su significatividad. Estos elementos lo relacionan con un aumento de la motivación. (MOTIVACIÓN)

3. La auto-reflexión cómo actividad que se realiza principalmente en las tareas que se les solicita una autoevaluación o después de recibir el feedback del docente. Uno de los aspectos que teníamos en cuenta era la auto-reflexión, la cual se da en pocos casos y menos de forma consciente. (AUTO-REFLEXIÓN)

4. El uso de los criterios de evaluación o rúbricas facilitadas por el docente al plantear la tarea a desarrollar. Estos les sirven de control de que se está realizando lo esperado y que hay aprendizaje. Respecto a las rúbricas hay diversidad de opiniones, desde los que consideran imprescindible su uso desde el inicio hasta los que consideran que su exceso de detalle en ocasiones les llega a limitar su creatividad y originalidad impidiendo mostrar su propia identidad. Otro aspecto mencionado es la necesidad que las rúbricas sean elaboradas en consenso entre docentes-discentes. (RECURSOS-HERRAMIENTAS)

5. La planificación les ayuda a regular el aprendizaje en el tiempo, en las subtareas y en el cumplimiento de los objetivos de la actividad. Nombran el uso de listas de priorización, temporalización y checklist. En el resto de las fases la autorregulación se da de modo más inconsciente. (PLANIFICACIÓN-ORGANIZACIÓN)

6. La retroacción entre compañeros (o entre iguales) se ha referenciado sobre todo en la fase de Planificación para comprender el enunciado del ejercicio o tarea (¿Hemos entendido todos lo mismo?) y antes de la entrega del producto final. Estas retroacciones dicen que se da habitualmente en conversaciones informales y porqué tienden a realizar los trabajos académicos en grupo por indicación del docente. (RETROACCIÓN-COMPAÑEROS).

Análisis de relatos de estudiantes universitarios sobre la autorregulación en experiencias de evaluación 
Hay tres elementos más que han aparecido a menudo en los relatos analizados, pero en menos intensidad: la capacidad de reorganización, la revisión continuada de los objetivos de aprendizaje y los factores emocionales que condicionan el esfuerzo, la dedicación, la constancia, la persistencia, la voluntariedad, etc.

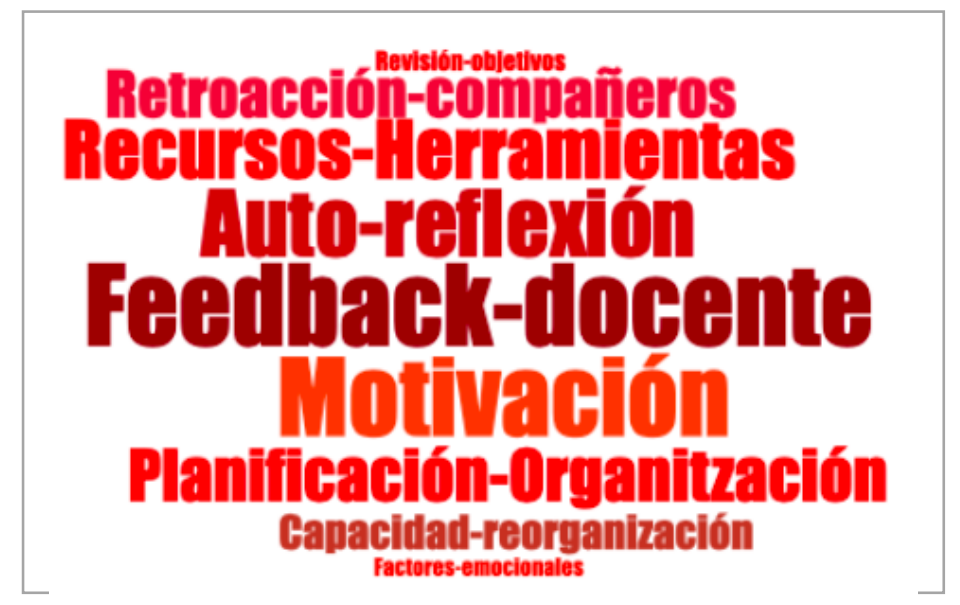

Figura 1. Grado de reiteración de elementos relacionados con la autorregulación del aprendizaje.

En los relatos citan 34 barreras para el correcto desarrollo de los trabajos y 16 estrategias que les han ayudado a conseguir exitosos resultados. Los estudiantes indican que mayoritariamente trabajan en grupo por lo que este término se repite constantemente en su discurso cómo un facilitador o cómo una barrera para la correcta autorregulación. Por otro lado, se ha observado que hay una preocupación en exceso por la calificación final que le otorgará el profesor (evaluación acreditativa).

\section{Conclusiones}

A lo largo de los relatos se aprecia que se dan distintas fases en el proceso de regulación académica, aunque pocos son los estudiantes que hacen referencia a ellas de forma explícita. De modo explícito se hace referencia a la relevancia de la evaluación formativa resaltando la importancia de la retroalimentación del docente, la retroacción de los compañeros (a menudo en formato de alerta y en pocas ocasiones de contenido), los instrumentos que facilitan la autoevaluación tales como el listado de criterios de evaluación (que al ser en muchos casos genéricos les sirve de checklist) o las rúbricas (que preferirían que fueran más consensuadas entre docentes y discentes). A partir de las prácticas identificadas, el siguiente paso es describir, implementar en la formación de los Grados objeto de estudio y analizar estrategias que potencien el desarrollo de procesos de autorregulación aplicables en la futura profesión educativa. 


\section{Referencias}

Andrade, H. L. (2010). Students as the definitive source of formative assessment: academic self-assessment and the self-regulation of learning. En Andrade, H. L. \& Cizek, G. J. (Eds.). Handbook of formative assessment (90-105). New York: Routledge.

Cano, E. (2014). Análisis de las investigaciones sobre feedback: aportes para su mejora en el marco del EEES. Bordón, 66(4), 9-24.

Fuentes, M. (2017). Evaluación y TIC. Material didáctico de La Universidad Internacional de Valencia: Máster en educación, tecnologías e innovación (TIC).

Hernández, A., \& Camargo, Á. (2017). Autorregulación del aprendizaje en la educación superior en Iberoamérica: una revisión sistemática. Revista Latinoamericana de Psicología, 49(2), 146-160

Ion, G., Sánchez-Martí, A. \& Agud-Morell, I. (2019) Giving or receiving feedback: which is more beneficial to students' learning? Assessment \& Evaluation in Higher Education, DOI: 10.1080/02602938.2018.1484881

López-Pastor V. M. \& Pérez-Pueyo, A. (coords.) (2017). Evaluación formativa y compartida en Educación: experiencias de éxito en todas las etapas educativas. León: Universidad de León.

Nicol, D. J. \& Macfarlane-Dick, D. (2006). Formative assessment and self-regulated learning: a model and sever principles of good feedback practice. Studies in Higher Education, 31(2), 199-218.

Panadero, E. (2017). A Review of Self-regulated Learning: Six Models and Four Directions for Research. Frontiers in Psychology, 8(422), 1-22.

Pintrich, P. R. (2000). 'The role of goal orientation in self-regulated learning' in M. Boekaerts, P. R. Pintrich, and M. Zeidner (eds.). Handbook of Self-regulation. San Diego, CA: AcademicPress.

Villardón, L. (2006). Evaluación del aprendizaje para promover el desarrollo de competencias. Educación Siglo XXI, 24, 57-76.

Zimmerman, B. J. (2008). Investigating self-regulation and motivation: historical background, methodological developments, and future prospects. American Educational Research Journal, 45(1), 166-183. 